\title{
Detecting the manipulation of digital clinical records in dental practice.
}

Díaz-Flores García, Victor, Labajo González, Elena, Santiago Sáez, Andrés y Perea Pérez, Bernardo.

Cita:

Díaz-Flores García, Victor, Labajo González, Elena, Santiago Sáez, Andrés y Perea Pérez, Bernardo (2017). Detecting the manipulation of digital clinical records in dental practice. Radiography, $x x x(x x x), 1-5$.

Dirección estable: https://www.aacademica.org/elenalabajogonzalez/39 ARK: https://n2t.net/ark:/13683/pcQr/hqv 


\title{
Detecting the manipulation of digital clinical records in dental practice
}

\author{
V. Díaz-Flores-García ${ }^{\text {a }}$, E. Labajo-González ${ }^{\text {b, * }}$, A. Santiago-Sáez ${ }^{\text {b }}$, B. Perea-Pérez ${ }^{\text {b }}$ \\ a School of Health Sciences, Universidad Europea de Madrid, Spain \\ ${ }^{\mathrm{b}}$ Legal and Forensic Medical School of Madrid, School of Medicine, Universidad Complutense de Madrid, Spain
}

\section{A R T I C L E I N F O}

\section{Article history:}

Received 31 December 2016

Received in revised form

13 April 2017

Accepted 11 May 2017

Available online xxx

\section{Keywords:}

Digital clinical records

Manipulation

Forgery

Dentistry

\begin{abstract}
A B S T R A C T
Introduction: Radiography provides many advantages in the diagnosis and management of dental conditions. However, dental X-ray images may be subject to manipulation with malicious intent using easily accessible computer software.

Methods: In this study, we sought to evaluate a dentist's ability to identify a manipulated dental X-ray images, when compared with the original, using a variant of the methodology described by Visser and Kruger. Sixty-six dentists were invited to participate and evaluate 20 intraoral dental X-ray images, 10 originals and 10 modified, manipulated using Adobe Photoshop to simulate fillings, root canal treatments, etc.

Results: Participating dentists were correct in identifying the manipulated image in 56\% of cases, $6 \%$ higher than by chance and $10 \%$ more than in the study by Visser and Kruger.

Conclusion: Malicious changes to dental X-ray images may go unnoticed even by experienced dentists. Professionals must be aware of the legal consequences of such changes. A system of detection/validation should be created for radiographic images.
\end{abstract}

๑ 2017 The College of Radiographers. Published by Elsevier Ltd. All rights reserved.

\section{Introduction}

Since 1982, when Mouyen ${ }^{1}$ introduced the first digital system for dental radiography, images acquired with this technique have improved in terms of quality and the resultant radiation dose to the patient.

Digital radiography presents several advantages, such as the ease of storage of the image, no loss of quality with time, elimination of film processing and the associated need for a waste removal service and the purchasing of chemicals. Digital acquisitions allow the creation of multiple copies of the X-ray image and it allows some visual parameters to be improved through computer manipulation of the image. ${ }^{2}$

Along with these advantages software has been developed which allows the photographic retouching of images. This allows anyone with a computer and an image modification program to be potentially able to modify a dental X-ray image and completely vary its original appearance according to their requirements.

\footnotetext{
* Corresponding author. Escuela de Medicina Legal, Departamento de Toxicología y Legislación Sanitaria, Facultad de Medicina (Pab. 7), Universidad Complutense de Madrid, Ciudad Universitaria S/N 28040, Madrid, Spain. Fax: +34 913941606

E-mail address: elabajo@med.ucm.es (E. Labajo-González).
}

Taking into account that any dental X-ray image exported as an image file can be susceptible to image manipulation, this manipulation can divided into two categories:

- Non-malicious: it helps make the image more visible to the human eye and is designed to assist the reader,

- Malicious: data are added to or removed from the original image with a view to misleading the reader.

Image manipulation generates a problem of legal security since there is no control over the manipulation of the dental X-ray image in the event of a potential legal claim over treatment.

If the patient or dentist does not have access to a previous conventional radiography (non-digital) then it may be difficult to validate a failed treatment presented on digital media. This is because the possibility manipulation is unknown and currently there are no standardized systems available for the verification of radiographic images.

Attempts to standardize safety protocols have been reported within the literature, but have not yet been implemented in clinical practice. ${ }^{3,4}$ This may be due to logistical difficulties but it has also been highlighted the ease in which an image can be modified, often without a dental professional. Detection of such manipulations is also difficult, as shown in the study by Visser and Kruger, ${ }^{5}$ who 
observed that in most cases dentists were unable recognize the modified images. Likewise, Tsang, ${ }^{6}$ with a more crude system, based on conventional scanned radiographs, managed to make insurance companies believe that teeth which had been treated with simple fillings had in fact undergone complex endodontic treatment with intraradicular posts and crowns.

It is necessary, therefore, to create a validation system for digital radiographic images which is simple use and that can be implemented without problems into routine clinical practice.

The aim of this study was to evaluate a dentist's ability to identify a manipulated dental X-ray image when compared with the original using a variant of the methodology reported by Visser and Kruger. ${ }^{5}$

\section{Materials and methods}

Sixty-six practicing dentists were selected, all with professional experience ranging from 2 to 20 years. No criteria were applied in terms of gender, age or knowledge of computers, thereby following the participant selection process described by Visser and Kruger. ${ }^{5}$ The following changes to Visser and Kruger's methodology were also made:

- The number of participants was increased from 39 to 66 .

- Ten pairs of dental X-ray images were given out, one original and one manipulated, thereby allowing the participants to compare the two.

- Participants in the study had no time limit for the evaluations and were not provided with any tools to assist in the identification of the manipulated image.

- Participating dentists received e-mail messages containing 20 paired dental X-ray images, 10 originals and 10 modified (on the basis of the originals) (Figs. 1-5).

All of the dental X-ray images were obtained from personal clinical records of the research team, having been taken using the following digital imaging equipment: GXS-700 ${ }^{\circledR}\left(\right.$ Gendex $\left.^{\circledR}\right)$ and VistaScan Mini ${ }^{\circledR}$ (Dürr Dental ${ }^{\circledR}$ ).

All dental X-ray images were saved as a JPG file (Joint Photographic Experts Group) so that the original image could be modified using Adobe Photoshop CS6 software ${ }^{\circledR}$. The modifications that were carried out consisted of adding bone to radiolucent areas, placing restorations in unfilled teeth or putting in place radioopaque materials which simulated root canal treatments. Identification of each dental X-ray image was subject to a coding process in order to maintain anonymity. Each dental X-ray image was assigned an identification code which complied with the ethical approval for the study.

Once participants had received the image bank they were permitted to use all of the tools they thought necessary to identify the real image. Decision were recorded in a table prepared using Google Docs (Google Inc. ${ }^{\circledR}$ ) and all participants remained blind to one an others classifications.

\section{Statistical analysis}

To determine the general percentage of correct answers and the probabilities determined by chance, the Binomial test was used as a test of deviations from a theoretically predicted distribution of observations in two categories. This approach was similar to the data analysis reported in the study by Visser and Kruger. ${ }^{5}$

\section{Results}

Using the Binomial coefficient or combinatorial number, the probabilities determined by chance were calculated (Table 1 ) for all
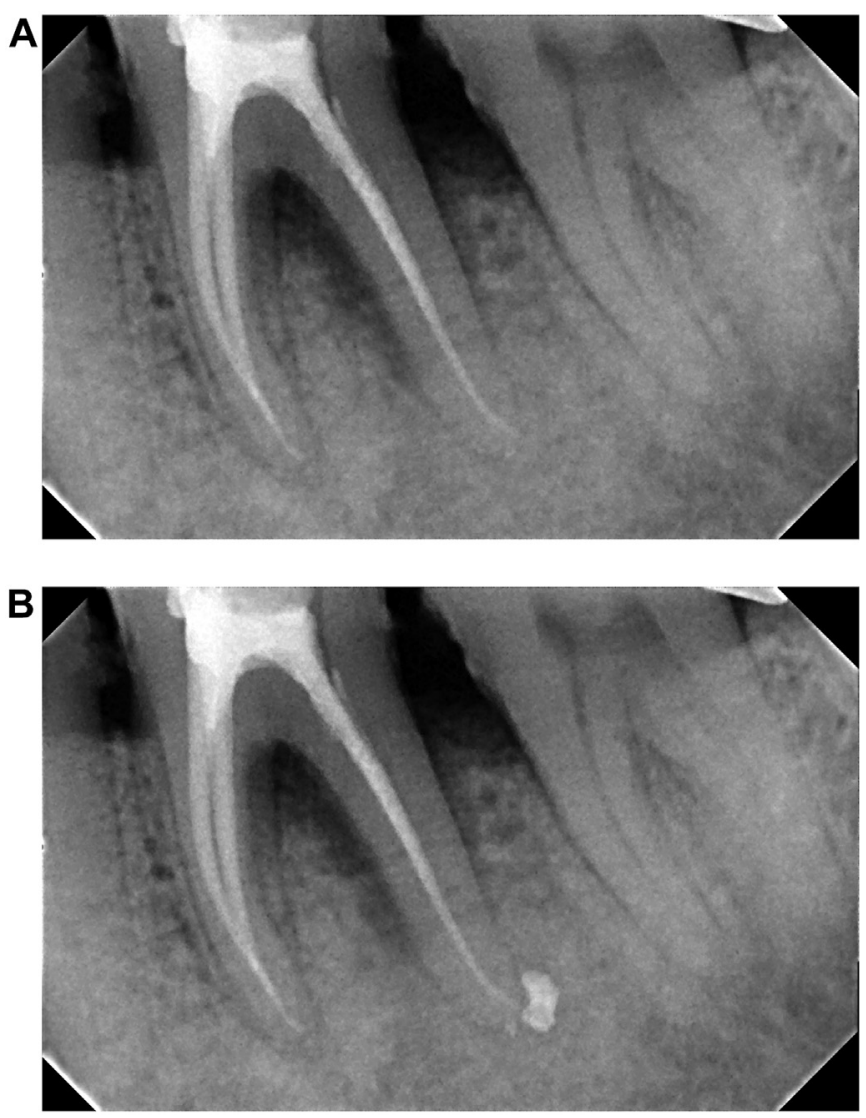

Figure 1. A. Sample image: non-manipulated. B. Sample image: manipulated, extrusion of dental cement to periapex added.

of the possible events in the sample space, or, the probability of selecting the correct type of radiograph (non-manipulated/ manipulated) zero, one, two times, etc. Likewise, the probability was calculated in our experiment for each of the events, using the Frequency Sampling or Objectivist Method or Bayesian Method that links the probability of A given B with the probability of B given A, or the probability of causal aspects given the observed effects (Table 2).

In order to determine the general percentage of correct answers $(\mathrm{P})$, bearing in mind the independent nature of the events, the number of favorable cases (correct answers) was divided by the number of possible cases, giving an overall result of correct answers equal to $56 \%$.

$$
P=\frac{\text { Correct answers }}{\text { All cases }}=\frac{371}{660}=0.56=56
$$

The study is made up of a series of Bernoulli experiments, and therefore the Binomial distribution can be used to make predictions.

\section{$\chi \sim \mathrm{B}(\mathrm{n}, \mathrm{p})$ in which $\mathrm{p}=0.56$}

In the results by radiograph, the breakdown of correct/incorrect answers was generally homogeneous, reaching close to $50 \%$. It was found that in 3 of the 10 pairs of radiographs which were analyzed, the percentage of incorrect answers was greater than the number of correct answers, and that the radiograph for which the correct option was selected most often did so among just $78 \%$ of the participants. 

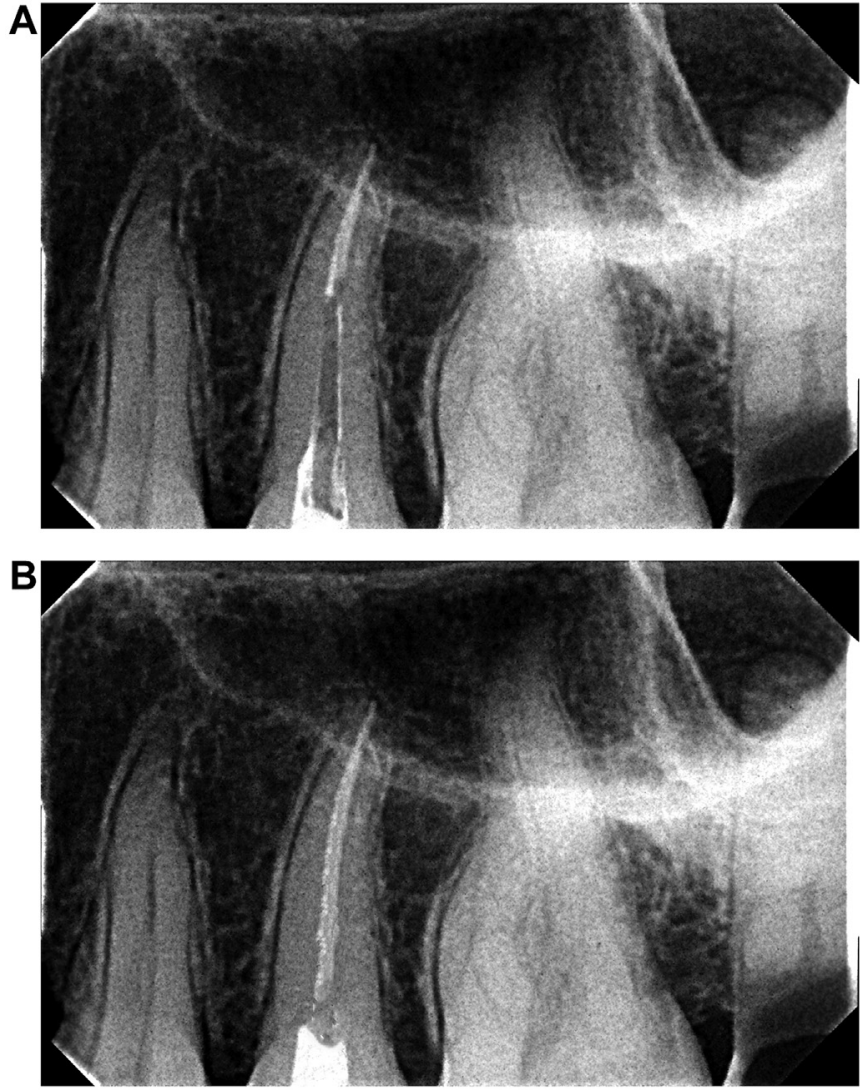

Figure 2. A. Sample image: non-manipulated, insufficient endodontic filling material. B. Sample image: manipulated.

\section{Discussion}

With the advent of digital radiology, the malicious manipulation of images has also been made possible. Using the programs which exist today, it is very simple to perform the manipulation of an Xray image. To remedy this problem, radiographic image processing programs began to generate files that could only be read by the program which generated them. However, it is simple to export an image into a conventional and editable format such as JPG, manipulate the image and then export it back into the radiography program and save it in the original program format. Fraudulent manipulation of images implies a problem of legal certainty, since it makes possible to fraud in dealings with medical insurance, for instance, for unrealized treatments when a manipulated radiograph is shown, can be considered as performed.

Calberson et al. ${ }^{8}$ made clear in 2008 how easy it is to manipulate a radiograph, describing methods for identifying such manipulation, and at present such manipulation can be prevented. In this sense, the studies on image manipulation in dentistry are few ${ }^{4-6}$ and practically all were carried out before the improvements which have taken place over the last decade in digital radiology. They are all based on the digitalization of radiographs using a negative scanner, with later processing through image editing programs. The study by Singbal et al., ${ }^{9}$ which dates back to 2010, continued using the classic methodology for these tasks: digitizing images using a photographic negative scanner and manipulating the radiograph with an image editing program. In this study, we point out the ease with which a radiograph can be manipulated.

Image manipulation has raised interest in recent years in the fields of forensics and biology, with frequent studies again
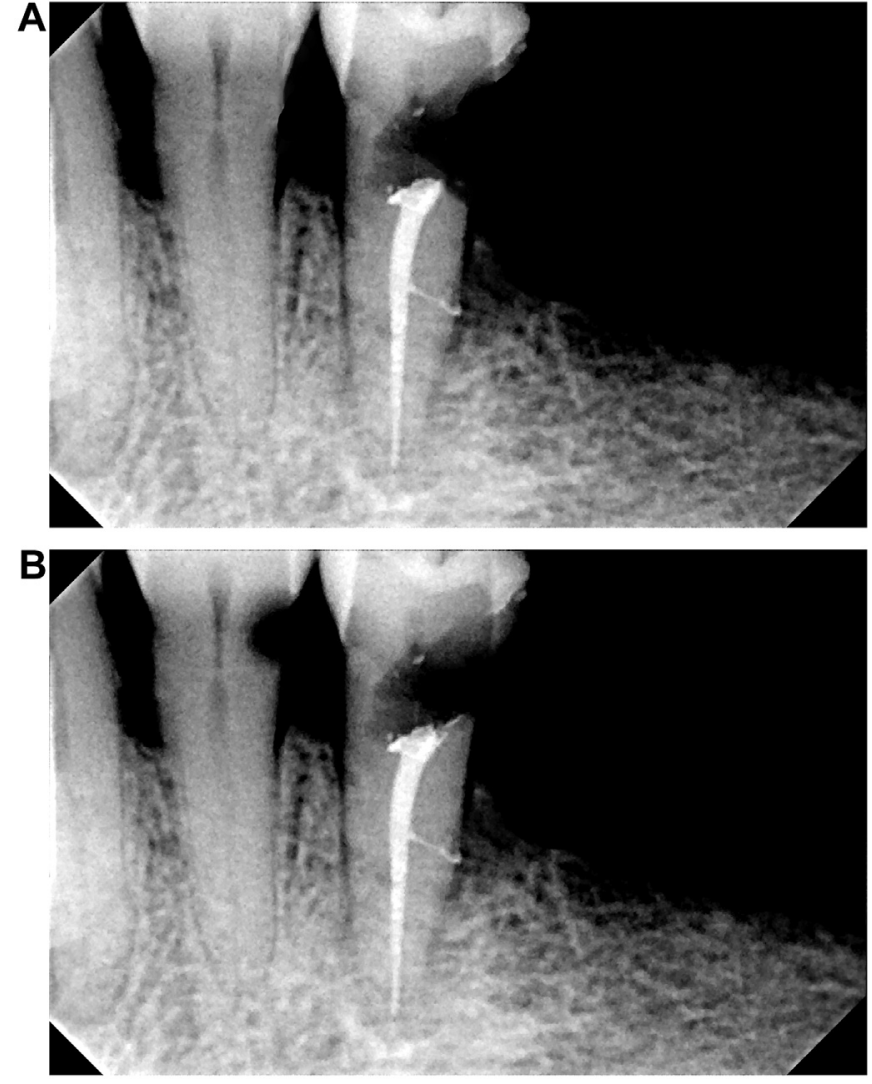

Figure 3. A. Sample image: non-manipulated. B. Sample image: manipulated, distal caries on premolar added.

demonstrating how easy it is for an X-ray image to be manipulated. ${ }^{10-12}$

Although all of the scientific literature on this topic is concerned with insisting upon this ease in manipulation, ${ }^{4-18}$ only two studies over the last 20 years have dealt with the dental professionals' skill in detecting manipulated radiographs. ${ }^{4,5}$ These studies make it obvious that visual inspection does not always make it possible to identify a modified (manipulated) X-ray image.

The results of our study show that the participants were able to select the original X-ray image correctly on 56\% of all occasions. This percentage differs only by $10 \%$ from that found in the study by Visser and Kruger (46\%). One must bear in mind that said study was completed in 1997, using image manipulation software with much less potential that the software which is in existence today. The improvement in these systems has made it possible for manipulation to become more difficult for the human eye to perceive. It should also be pointed out that, in the prior study, the participants did not have the original and modified X-ray images available for comparison, which may help explain the low percentage of correct answers. The fact that the difference between the two results is just $10 \%$, though the participant had more time, means and opportunity to make comparisons, leads us to believe that detecting modified radiographs may be impossible to achieve in the future.

The main limitations of the present study are related to the number of participants as well as the number of image pairs used, which undoubtedly conditions the results obtained. In future studies, it is intended to expand the sample in both cases. Regardless it is significant that, in this study, no participating dentist was able to identify each and every one of the original X-ray images (as occurred in the study by Visser and Kruger), even 

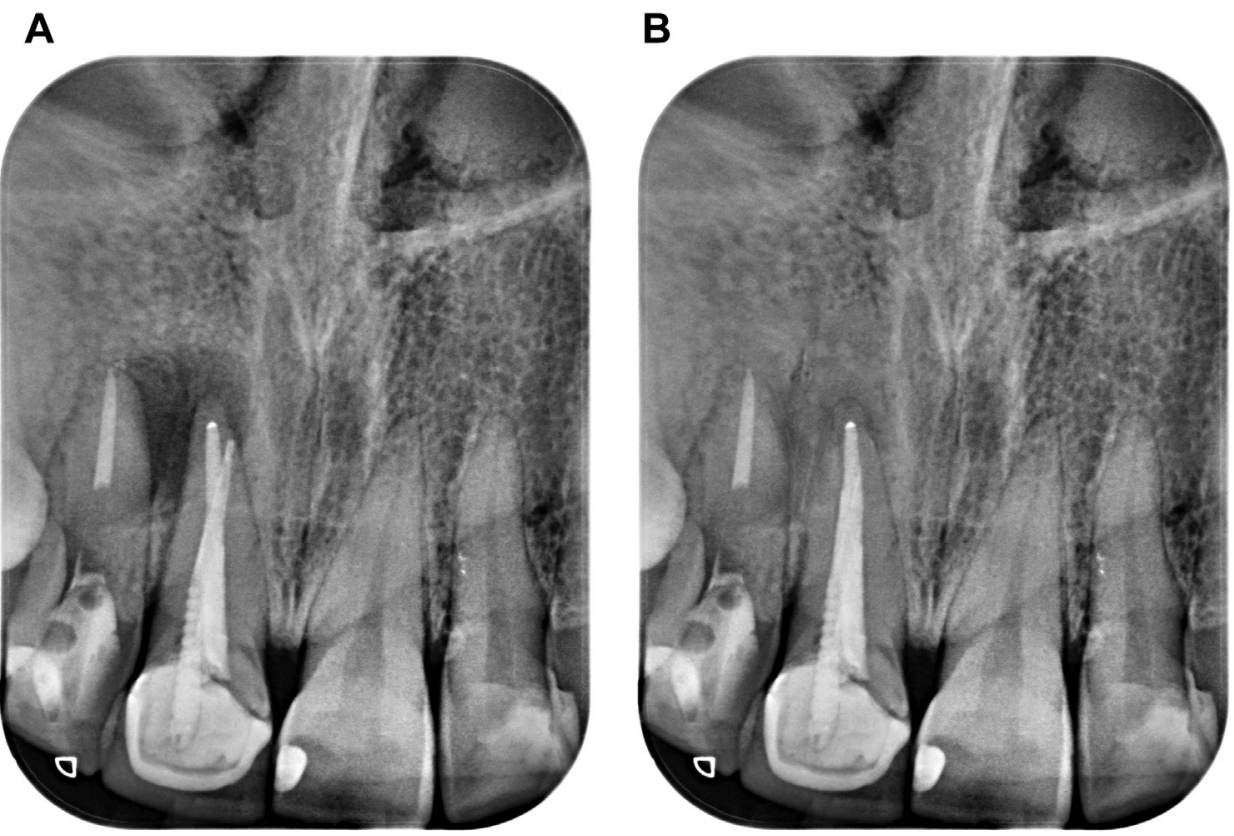

Figure 4. A. Sample image: non-manipulated, periapical radiolucent lesion. B. Sample image: manipulated.

A

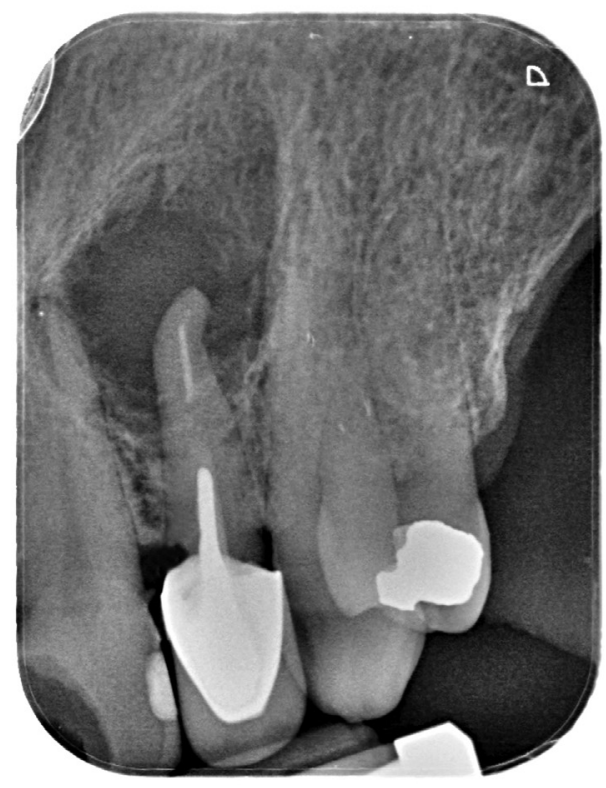

B

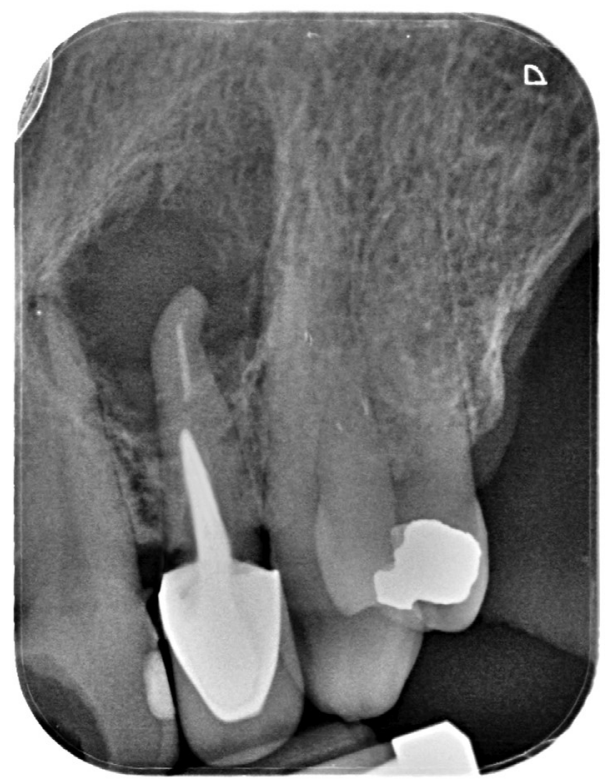

Figure 5. A. Sample image: non-manipulated, insufficient length bolt. B. Sample image: manipulated.

though there was no time limit on analyzing each image. This statistical analysis does not bear in mind the years of clinical experience or familiarity with the use of image enhancement programs. All of this means that, within the parameters of our study, the difference in selecting the authentic X-ray images differs by just $6 \%$ when comparing a dental professional and a person with no clinical experience. In other words, an inexperienced observer would, in general, be able to choose the correct type of image (nonmanipulated/manipulated) without having any dental training at all. Only 6\% more correct answers were achieved by asking experienced dentists to examine the images.
Therefore, it is necessary to create a system for the detection of manipulated X-ray images. As an example, Lien ${ }^{18}$ proposed a system for "signing" X-ray images with a digital certificate in 2010. This system would allow dentists to remain accountable for the X-ray images they take and for their handling. Despite the time which has elapsed since the publication of that article, no digital radiographic system yet includes the option of signing radiographs with a digital certificate. Implementing a method for validation or identification of radiographic tests would make it possible to increase legal security for both patients and dentists. 
Table 1

Binomial distribution of the probability of correct answers.

\begin{tabular}{lllll}
\hline $\begin{array}{l}\text { Correct } \\
\text { answers } \\
\text { ways } \\
\text { to get correct } \\
\text { answer }\end{array}$ & $\begin{array}{l}\text { Random } \\
\text { probability }\end{array}$ & $\begin{array}{l}\text { Number of } \\
\text { dentists vs. } \\
\text { number of } \\
\text { correct } \\
\text { answers }\end{array}$ & $\begin{array}{l}\text { Relative } \\
\text { frequency }\end{array}$ \\
\hline 0 & 1 & $5.412544 \times 10^{-6}$ & 0 & 0 \\
1 & 100 & $5.412544 \times 10^{-4}$ & 1 & 0.015151 \\
2 & 2025 & 0.010960 & 2 & 0.030303 \\
3 & 14,400 & 0.077940 & 3 & 0.045454 \\
4 & 44,100 & 0.238693 & 11 & 0.166666 \\
5 & 63,504 & 0.343718 & 11 & 0.166666 \\
6 & 44,100 & 0.238693 & 17 & 0.257575 \\
7 & 14,400 & 0.077940 & 14 & 0.212121 \\
8 & 2025 & 0.010960 & 5 & 0.075757 \\
9 & 100 & $5.412544 \times 10^{-4}$ & 2 & 0.030303 \\
10 & 1 & $5.412544 \times 10^{-6}$ & 0 & $\mathbf{1}$ \\
\hline
\end{tabular}

Table 2

Percentage of correct/incorrect answers by image set

\begin{tabular}{lll}
\hline Image set & $\begin{array}{l}\text { \% Correct } \\
\text { answers }\end{array}$ & $\begin{array}{l}\text { \% Incorrect } \\
\text { answers }\end{array}$ \\
\hline GROUP 1 & 53 & 47 \\
GROUP 2 & 53 & 47 \\
GROUP 3 & 78 & 22 \\
GROUP 4 & 59 & 41 \\
GROUP 5 & 46 & 54 \\
GROUP 6 & 34 & 66 \\
GROUP 7 & 71 & 29 \\
GROUP 8 & 74 & 26 \\
GROUP 9 & 57 & 43 \\
GROUP 10 & 37 & 63 \\
\hline
\end{tabular}

\section{Conclusion}

The participating dentist was correct in $56 \%$ of cases, on $6 \%$ higher than by chance, and $10 \%$ more than the result found in the study by Visser and Kruger; therefore we can conclude that new image manipulation systems make it possible to carry out malicious changes in X-ray images which are undetectable and a system of detection/validation must be created for radiographic images.

\section{Conflict of interest statement}

None.

\section{References}

1. Mouyen F, Benz C, Sonnabend E, Lodter J. Presentation and physical evaluation of RadioVisioGraphy. Oral Surg Oral Med Oral Pathol 1989;68:238-42.

2. Farman AG, Farman TT. A status report on digital imaging for dentistry. Oral Radiol 2004;20:9-14.

3. Jones GA, Behrents RG, Bailey GP. Legal considerations for digitized images. Gen Dent 1996;44(3):242-4.

4. Boscolo FN, Almeida SM, Haiter Neto F, Oliveira AE, Tuji FM. Fraudulent use of radiographic images. J Forensic Odontostomatol 2002;20:25-30.

5. Visser H, Kruger W. Can dentists recognize manipulated digital radiographs? Dentomaxillofac Radiol 1997;26:67-9.

6. Tsang A, Sweet D, Wood RE. Potential for fraudulent use of digital radiography. J Am Dent Assoc 1999;130:1325-9.

7. Van der Stelt PF. Better imaging: the advantages of digital radiography. J Am Dent Assoc 2008;139:7S-13S.

8. Calberson FL, Hommez GM, De Moor RJ. Fraudulent use of digital radiography: methods to detect and protect digital radiographs. J Endod 2008;34(5):530-6.

9. Singbal KP, Chhabra N, Madan B. Digital imagery: reality or fakery. Int J Contemp Dent 2010;1(3):93-8.

10. Pearson H. Forensic software traces tweaks to images. Nature 2006;439(7076): $520-1$.

11. Rossner M, Yamada KM. What's in a picture? The temptation of image manipulation. J Cell Biol 2004;166(1):11-5.

12. Du Chesne A, Benthaus S, Brinkmann B. Manipulated radiographic material-capability and risk for the forensic consultant? Int J Leg Med 1999;112(5): 329-32.

13. Pass B, Furkart AJ, Dove SB, McDavid WD, Gregson PH. 6-bit and 8-bit digital radiography for detecting simulated periodontal lesions. Oral Surg Oral Med Oral Pathol 1994;77:406-11.

14. Bruder GA, Casale J, Goren A, Friedman S. Alteration of computer dental radiography images. J Endod 1999;25(4):275-6.

15. Güneri P, Akdeniz BG. Fraudulent management of digital endodontic images, Int Endod J 2004:37(3):214-20.

16. Sparavigna AC. An imaging processing approach based on GNU image manipulation program GIMP to the panoramic radiography. Int $J$ Sci 2015;4(05):57-67.

17. Jin-Woo C, Won-Jeong H, Eun-Kyung K. Imaging enhancement of digital periapical radiographs according to diagnostic task. Imaging Sci Dent 2014;44: $31-5$.

18. Lien CY, Kao T, Hsiao CH, Keng CI. A software-embedded method of security protection applied in indirect imaging in dentistry. J Med Biol Eng 2010;30(4): 203-7. 